\title{
$Q$-NORM INEQUALITIES FOR SEQUENCES OF HILBERT SPACE OPERATORS
}

\section{S. S. Dragomir, M. S. Moslehian and J. SÁndor}

Abstract. We give some inequalities related to a large class of operator norms, the so-called $Q$ norms, for a (not necessary commutative) family of bounded linear operators acting on a Hilbert space that are related to the classical Schwarz inequality. Applications for vector inequalities are also provided.

Mathematics subject classification (2000): 47A05, 47A12.

Keywords and phrases: Bounded linear operators, Hilbert spaces, Schwarz inequality, Commuting operators.

\section{REFERENCES}

[1] R. Bhatia, Matrix Analysis, Springer-Verlag, New York, 1997.

[2] D. E. Day Kin And C. J. Eliezer, Generalization of Hölder and Minkowski's inequality, Proc. Cambridge Phil. Soc., 64 (1968), 1023-1027.

[3] S. S. Dragomir, Some Schwarz type inequalities for sequences of operators in Hilbert spaces, Bull. Austral. Math. Soc., 73 (2006), 17-26.

[4] S. S. DRAGOMIR, Norm inequalities for sequences of operators related to the Schwarz inequality, J. Inequal. Pure Appl. Math., 7, 3 (97) (2006), 10 pp.

[5] D. S. Mitrinović, Analytic inequalities, Springer Verlag, 1970.

[6] B. Simon, Trace Ideals and their Applications, Cambridge University Press, Cambridge, 1979. 